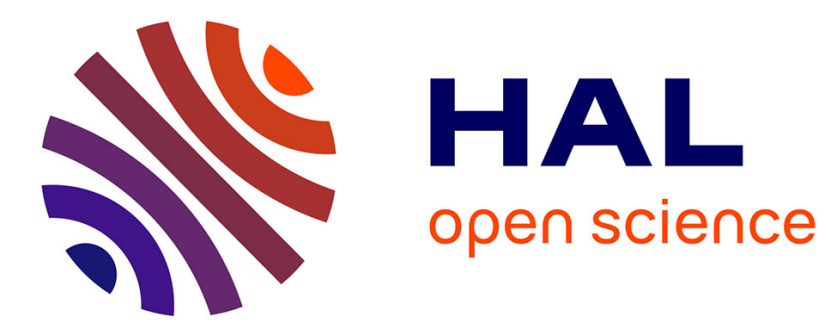

\title{
Études cam. IV. Une relation ibérique du Campa en 1595
}

Pierre-Yves Manguin

\section{To cite this version:}

Pierre-Yves Manguin. Études cam. IV. Une relation ibérique du Campa en 1595. Bulletin de l'Ecole française d'Extrême-Orient, 1981, 70 (1), pp.253 - 269. 10.3406/befeo.1981.3379 . halshs-02509294

\section{HAL Id: halshs-02509294 \\ https://shs.hal.science/halshs-02509294}

Submitted on 16 Mar 2020

HAL is a multi-disciplinary open access archive for the deposit and dissemination of scientific research documents, whether they are published or not. The documents may come from teaching and research institutions in France or abroad, or from public or private research centers.
L'archive ouverte pluridisciplinaire HAL, est destinée au dépôt et à la diffusion de documents scientifiques de niveau recherche, publiés ou non, émanant des établissements d'enseignement et de recherche français ou étrangers, des laboratoires publics ou privés. 


\section{Études cam. IV. Une relation ibérique du Campa en 1595}

Pierre-Yves Manguin

\section{Citer ce document / Cite this document :}

Manguin Pierre-Yves. Études cam. IV. Une relation ibérique du Campa en 1595. In: Bulletin de I'Ecole française d'ExtrêmeOrient. Tome 70, 1981. pp. 253-269;

doi : https://doi.org/10.3406/befeo.1981.3379

https://www.persee.fr/doc/befeo_0336-1519_1981_num_70_1_3379

Fichier pdf généré le 08/02/2019 


\title{
ETUDES CAM
}

\section{UNE RELATION IBÉRIQUE DU CAMPĀ EN 1595}

PAR

\author{
Pierre-Yves MANGUiN
}

\begin{abstract}
La "Relation des affaires du Campā " qui est présentée ici n'est pas en soi un texte de grande valeur, tant par sa brièveté que par certaines inexactitudes d'ordre géographique. Mais l'extrême rareté des sources de première main concernant spécifiquement le Campā après la chute de sa capitale Vijaya aux mains des Viêtnamiens en 1471 en fait un document quasi unique. Pour des raisons multiples qu'il est difficile d'apprécier avec précision - déclin, importance économique toute relative, pays peu hospitalier —, le Campā fait peu parler de lui dans les sources ibériques $\mathrm{du} \mathrm{XvI}^{\mathrm{e}}$ siècle. Après les deux descriptions succinctes qu'en ont données les Portugais Tomé Pires et Duarte Barbosa dans la deuxième décennie de ce siècle ${ }^{1}$, il n'en est fait que très occasionnellement mention jusqu'à la fin du siècle, lorsque apparaît la documentation contemporaine et de même origine que le texte édité ici.

La seule exception, notoire, est à ma connaissance une description tout aussi brève publiée et traduite en anglais par C. R. Boxer et qui est extraite d'un codex manuscrit en sa possession, compilé à Manille dans la dernière décennie $d u x{ }^{e}{ }^{e}$ siècle $e^{2}$ Ce codex, rédigé en espagnol et illustré par un artiste qui semble bien avoir été chinois, regroupe nombre de descriptions disparates de diverses régions de l'Asie du Sud-Est, dont certaines, si ce n'est toutes, avaient pour originaux des textes portugais aujourd'hui disparus. Comme le dit bien C. R. Boxer, cette
\end{abstract}

(1) Cortesão a édité la Suma Oriental (1944; vol. I : 112-115 pour la traduction anglaise, vol. II : 390392 pour l'original portugais $\rangle$. Pour une traduction française annotée du seul chapitre de Pires sur le Campā, cf. Manguin 1972 : 39-42. Le texte de Barbosa a été édité par Dames 1918 (vol. II : 208). On en trouvera le texte portugais dans Colleçáo de noticias para a hislória e geographia das nações ullramarinas (2e éd.), t. II, Lisboa $1867: 237-388$ (p. 373 pour la citation sur le Campā).

(2) Le texte de cette Relacion de las costumbres del Reyno de Champa est donné dans Boxer 1970. Le codex d'où est extrait cette relation est décrit en détail dans Boxer 1950. 
autre relation "gives the impression of having been included in the codex as an afterthought ". Comme il le fait encore remarquer, l'Islam n'y est pas mentionné et seules des pratiques śivaïtes y sont décrites (comme la satī de la veuve). Les présomptions sont certes minces, mais il semble qu'on puisse en déduire que cette relation, dont la source pourrait avoir été différente de celle des autres textes de ce codex, est antéricure à la compilation de ce dernier et, par conséquent, à la date de 1595 que lui attribue Boxer. Elle aurait été écrite alors que l'Islam n'avait encore fait que peu ou pas de progrès au Campā. Son implantation certaine n'est attestée qu'à partir de 1595, mais il faut rappeler qu'entre 1518 et cette date, on ne connaît aucun autrc document faisant état des religions pratiquées au Campāi . Cette relation a été écrite à Luzon - comme l'attestent ses premières lignes -..., donc après la prise de Manille en 1564. C'est par conséquent entre ces deux dates de 1564 et 1595 qu'elle aurait été écrite et on peut présumer que les premières conversions notables à l'Islam datent d'après 1564 (ce que j'avais omis de noter dans ma précédente étude sur l'introduction de l'Islam au Campā). Cette description, en dépit de certaines exagérations évidentes, fournit d'importants renseignements sur les rites et les festivités du pays cam et complète en cela le texte présenté ici qui est pour sa part très largement consacré à des considérations stratégiques et économiques, bien qu'on y trouve quelques mentions fort intéressantes sur les mœurs et les religions.

Pour sa bonne compréhension, cette "Relation des affaires du Campā", datée de 1595, doit être replacée dans son contexte. A la fin $\mathrm{du} \mathrm{XvI}^{\mathrm{e}}$ siècle, alors que le Portugal est tombé depuis 1580 sous la domination castillane, naissent aux Philippines sous la pression du gouverneur Luiz Pérez Dasmariñas et de l'ordre dominicain, et à Malaka sous l'égide d'un évêque quelque peu mégalomane du nom de João Ribeiro Gaio, bon nombre de projets de conquête des pays de l'Asic du Sud-Est et même de la Chine ${ }^{2}$. Aucun de ces projets ne verra le jour, si l'on excepte celui du Cambodge, où quelques aventuriers portugais et espagnols profitent des crises dynastiques des années 1596-1599 pour tenter sans succès d'en faire bénéficier la couronne de Castille. Mais du moins ces projets nous vaudront-ils une abondante littérature descriptive sur la région, la documentation amassée dans ce but tant à Malaka qu'à Manille ayant été le plus souvent d'excellente qualité, bénéficiant de la longue expérience des Portugais dans les pays et les réseaux commerciaux du pourtour de la Mer de Chine.

Cette "Relation des affaires du Campā" a donc été écrite pour justifier d'une éventuelle conquête de ce pays par des troupes espagnoles. Cela explique qu'elle s'attarde à décrire la position stratégique dont jouit le Campā sur la route obligatoire de la traversée de la Mer de Chine méridionale et le peu de moyens qu'il a de se défendre. Les rites "abominables" qui y sont pratiqués par un roi encore hindouïste, s'ils ne sont mentionnés qu'en passant dans ce texte, ont servi cette même

(1) Manguin $1979: 269$ sq.

(2) Ces projets et leurs motivations sont décrits en détail dans Boxer 1969. 
année 1595 de prétexte à une assemblée de théologiens réunie à Manille pour justifier cette conquête et punir le "tyrannique roi du Champa (...) d'une guerre de feu et de sangi ". Le fait, enfin, que ce même roi fasse alors preuve d'une attitude parfaitement libérale vis-à-vis de l'Islam, allant jusqu'à favoriser son implantation, est ressenti à Manille comme une incitation à la guerre sainte. En comparaison, les nombreux produits d'exportation du pays ne paraissent signalés que par souci d'être exhaustif. Si d'autres sources ne venaient nous confirmer l'exactitude de ces trois grandes têtes de chapitre (position clef sur une route maritime essentielle, rites hindouïstes et islamisation), on serait tenté de douter de leur bien-fondé, eu égard au but avoué que poursuit le commanditaire de cette "Relation " et à l'inexactitude de certains détails d'ordre géographique. Mais il faut dire que ses auteurs sont certainement les Européens les mieux informés de l'époque sur cette région. Ils sont loin, en effet, d'être des inconnus. Diogo Veloso qui - dit le colophon a pris ses informations auprès des Cam du Cambodge, où il a vécu neuf années durant, est cet aventurier portugais qui a été à l'origine de l'expédition espagnole dans ce pays. "Fils adoptif " du souverain khmer selon les chroniques cambodgiennes, il connaissait indubitablement bien la région. Blaz Ruiz de Mernán Gonzáles, espagnol installé aux Philippines, était comme Diogo Veloso au nombre des favoris du souverain khmer. Son témoignage découle de son expérience vécue : il avait en effet été fait prisonnier par le roi du Campā en 1592, alors qu'il s'était embarqué à bord du navire de Gregorio Vargas Machuca, autre témoin visuel et cosignataire de ce texte. Peut-être le Portugais Francisco Sagredo était-il lui aussi à bord de ce navire, puisqu'il témoigne de visu. Toujours est-il qu'avec son compatriote Pantaleão Carneiro on le retrouve parmi les aventuriers qui prennent part aux événements du Cambodge dans les années 1590. Diego de Chaves Cañizares, enfin, semble bien avoir été un haut fonctionnaire de l'administration des Philippines'. C'est donc, on le voit, à bonne source qu'à été puisée cette "Relation des affaires du Campā".

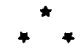 \\ Relation des affaires du Campā}

En exécution de l'ordre que Votre Seigneuric m'a donné de rédiger une relation sur l'état du royaume de Campā et de ce qui s'y trouve, j'affirme :

Que la capitale dudit royaume, où réside le roi, se trouve au bord de la mer, sur un cap qui s'avance loin en mer. Elle est située par $10^{\circ}$ [de 21 sq.).

(1) Les opinions de ces dix-huit théologiens ont été traduites par Cabaton (1914-16 :

(2) Sur tous ces personnages, on se reportera au résumé très clair des événements du Cambodge, puisé aux meilleures sources, qui est donné par Groslier et Boxer (1958 : 34 sq.), ainsi qu'aux divers travaux qui s'y trouvent cités. 
latitude nord]. Au nord de ce cap et de cette ville, la côte s'étend pendant 40 lieues jusqu'au royaume de Cochinchine, voisin septentrional du Campā; vers l'ouest de ce cap et de cette ville, la côte s'étend sur 60 lieues jusqu'aux confins du Cambodge'.

Il y a un détroit de six lieues de large entre ce cap et cette ville et des hauts-fonds qui longent la côte pendant 200 lieues depuis le nord. Leur extrémité [méridionale] se trouve à la hauteur des dits cap et ville du Campā et ils commencent en face de l'ile de Hainan, sur la côte de Chine $^{2}$. Le possesseur de ce site et de cette pointe serait le maitre de cette mer toute entière, car au nord de ce détroit sont situés les royaumes de Cochinchine, de la grande Chine et du Japon; à son sud, se trouvent les royaumes du Cambodge, du Siam, de Patani, de Pahang, de Johor, d'Indragiri, de Sunda, de Sumatra, de Solor, de Timor, les iles de Banda, la grande Java, Brunei, Malaka et toutes les régions des Indes Orientales. Tous ces royaumes situés au sud de ce détroit et de cette pointe ont des relations commerciales avec ceux qui se trouvent à son nord et réciproquement; il s'échange d'une région à l'autre de grandes et opulentes marchandises, en un très riche négoce. Ce détroit constitue un passage obligatoire pour naviguer de l'une à l'autre de ces régions, dans un sens comme dans l'autre lors des diverses saisons, car il n'existe pas d'autre route. On est forcé de passer à raser la terre ferme et le cap, car la côte y est libre de dangers et on peut y jeter la sonde ${ }^{3}$, et pour fuir les hautsfonds et les forts courants qui s'y trouvent. Les nefs des Portugais empruntent de même le dit détroit lorsqu'elles vont de l'Inde à la Chine et au Japon, et elles repassent ici-même au retour car, comme je l'ai dit,

(1) En ce qui concerne la localisation de la capitale et l'extension territoriale du Campā, voir infra, appendice $A$.

(2) La croyance en ces immenses récifs est encore générale au xvie siècle, comme elle l'était pour les navigateurs orientaux des siècles précédents : les routiers et la cartographie portugais des $\mathrm{XVI}^{\mathrm{e}}$ et $\mathrm{XVII}^{\mathrm{e}}$ siècles décrivent avec force détails et représentent cette barrière imaginaire de hauts-fonds qu'ils nomment $O$ Parcel ("Le Récif ", d'où le toponyme moderne : Paracels). Elle se serait étendue des véritables îles et récifs des Paracels (Hoàng sa), au sud de Hainan, jusqu'aux environs de Pulo Cécir de Mer et des iles et récifs qui l'entourent. Ce n'est qu'à la fin du xvir ${ }^{e}$ siècle que l'extension exacte de ces récifs sera connue. Jusque-là, les navigateurs de toutes nationalités ont toujours considéré qu'ils formaient un chenal obligatoire au long des côtes de la péninsule indochinoise : c'est donc en vue de celles-ci, et à portée des flottes du Campã disposées en des points stratégiques comme le cap Padaran (Mūi Dĩnh) ou, auparavant, le cap Varella (Núi Đá Bia) que passent tous les navires qui traversent du nord au sud et inversement la mer de Chine méridionale. Un Jésuite portugais, en 1686 décrit en termes imagés cette situation : "La moindre risée pousse les marins qui longent ces côtes dans ses baies [du Campā]. Celui qui vient de l'Inde y comptera en passant les branches de ses arbres; celui qui va au Cambodge y verra les gens ramasser du bois dans ses forêts ; celui qui se rend au Siam apercevra les torrents qui dévalent de ses montagnes. Personne ne peut passer le Champa sans voir et entendre les oiseaux dans ses arbres, personne ne peut aller au Tonkin sans longer ses cótes, sans apercevoir ses villages. Tous lui paient leur passage " (Ms. de la Bibliothèque d'Ajuda, à Lisbonne, collection Jesuitas na Asia, 49-V-19 : fo $.853 v^{\circ}$.) Les Cam semblent bien avoir été très tôt tentés ainsi de contrôler cette route maritime essentielle (d'où le qualificatif de "pirates "qui leur est souvent associé). Voir à ce propos Manguin 1972: 72 n. 5, 171-175 et carte 3; Manguin 1976 a.

(3) C'est-à-dire que les fonds ne sont pas trop grands et que les pilotes peuvent utiliser la sonde pour s'aider dans la détermination de leur position : les routiers de l'époque donnent en effet de nombreuses mesures de profondeur le long de cette route (Manguin 1972:65 sq.). 
il n'y a pas d'autre route. Ce leur serait d'une grande commodité et sécurité pour leurs voyages que Votre Majesté possède ce site, car il se trouve presque à mi-chemin entre Malaka et la Chine et ils pourront s'y procurer des rafraichissements et $y$ hiverner. Ce qu'ils ne font par crainte des Cam; et ils prennent ainsi parfois de grands risques, à l'aller comme au retour, car ils font parfois leurs voyages tardivement, et en hiver.

Le Campā est un pays plat et sec et très agréable, raisonnablement peuplé; son ciel est fort bon et le climat y est sain ${ }^{1}$. Il s'y trouve de nombreuses marchandises, dont du tissu de coton et de soie, de la cire et $\mathrm{du}$ fer et il y a beaucoup de montagnes productrices d'ébène, des éléphants, dont on tire beaucoup d'ivoire, des rhinocéros, dont on tire bien des choses'. Il s'y trouve un bois que l'on nomme calanba, aux vertus multiples; il est noir et huileux et de beaucoup de valeur ${ }^{3}$. On y trouve aussi beaucoup de bois fort propre à la construction des navires et le pays est très propice pour ce faire, car tous les naturels sont menuisiers et forgerons et qu'il y a comme je l'ai dit beaucoup de fer 4 . L'avitaillement y est abondant : riz, vin, vaches, poules, cerfs, porcs, chèvres, buffles, lièvres, perdrix, lapins, tourterelles, paons royaux et beaucoup de poissons ${ }^{5}$; il s'y trouve beaucoup de chevaux dont ils font

(1) Le Campā n'est un pays plat que dans sa partie méridionale, au sud du cap Padaran. Son climat est bien relativement sec puisque toute la plaine littorale qui constitue au xvi ${ }^{e}$ siècle ce pays reçoit moins de $1500 \mathrm{~mm}$ d'eau (789 mm à Phan-rang; Sion 1929 : 437).

(2) Tous ces produits sont parmi ceux dont la liste est donnée dans le Đại-nam nhât thông chi (éd. Sài-gỏn 1965, quyển 12 : p. 71-74).

(3) Du malais kelembak. C'est le bois d'aigle, exportation la plus recherchée du Campā, qui produit celui de meilleure qualité. Il est utilisé pour ses valeurs aromatiques. Voir à ce propos Manguin 1972 : 244-248 et, pour plus de détails, les références qui y sont citées.

(4) Les espèces de bois sont nombreuses, comme l'atteste la liste déjà citée du Đại-nam nhât thông chi. Les Portugais de Macau s'arrêtaient souvent dans la région pour y construire des navires. Il est intéressant de noter qu'à la fin du xvie siècle les charpentiers cam gardent leur renom. On sait par ailleurs qu'à cette même époque les Cam construisaient encore des navires avec lesquels ils fréquentaient divers ports de l'Asie du Sud-Est et de Chine (Grimm 1961 : 10, qui cite le chapitre du Ming shi sur le Siam; Manguin 1979:276-277). D'Après de Mannevillette amirme qu'en 1720 " par mer, les Loyes [les Cam] l'emportent [sur les Viêtnamiens] ; leurs galères sont mieux construites * (Le Neplune Oriental, Paris 1775 : 148). En 1740, les Jésuites diront encore du Campã que " ses habitants sont des gens de mer et en ont toutes les qualités " (Lettres édifiantes el curieuses sur la visile apostolique de M. de la Baume (...), Venise 1746 : 201).

(5) L'abondance du poisson sur les còtes de la péninsule indochinoise a toujours prappé les imaginations. Dès les origines du Campā, la région est peuplée de populations ichtyophages, dont l'exislence est notée par les sources chinoises comme par Ptolémée (Stein 1947:63-64:. Edrisi, au xire siècle, nole que "la mer de Campā nourrit une grande quantité de poissons, grands et petits * (Ferrand 1913-14, vol. I : 189). On rappellera encore qu'Odoric de Pordenone, au xive siècle, signale aussi la très grande abondance des poissons, qui viennent faire révérence au roi cam (Yule et Cordier 1913-16, II : 164-165). Cela n'est pas sans rappeler la cérémonie décrite dans la relation du xvie siècle publiée par Boxer $(1970: 38,41)$ : à la saison de la pêche, c'est le roi qui, le premier, jette cérémoniellement son fllet à la mer. En 1720 encore, "leurs bateaux en forme de tartane leur servent à la pêche, dont ils tirent beaucoup de poisson " (D'Après de Mannevillette, Le Neptune Oriental, Paris $1775: 148$ ). C'est donc au moins jusque dans la première moitié du $x^{\prime}{ }^{e}{ }^{e}$ siècle que les Cam maintiennent des rapports privilégiés avec la mer (voir aussi la note précédente). Si leur commerce extérieur a dù cesser à la fin du $\mathrm{Xvi}^{e}$ siècle lorsque leurs derniers ports sont passés sous contrôle viêtnamien, leurs activités piscicoles se sont prolongées au-delà. 
usage, et de même des éléphants dont un grand nombre sont apprivoisés. Ils ont beaucoup de chars à bœufs et à buffles ${ }^{1}$. Les habitants ne peuvent tuer ni manger de vache, car c'est une loi entre eux, et c'est la raison pour laquelle il y en a beaucoup 2 .

Le peuple de ce royaume est vêtu d'habits de coton et de soie; ils portent les cheveux longs noués sur le dessus de la tête ${ }^{3}$. Ils sont faibles et pusillanimes, ennemis des armes, vicieux, amis des fêtes et des passetemps. La raison de ces vices et de cette négligence tient au fait que ce site est si puissant et qu'il est un passage aussi obligé pour que tous ces royaumes puissent effectuer leur commerce; car ils sortent en mer pour voler tous les navires faibles ou qui ne sont pas sur leurs gardes lorsqu'ils passent par-là et ils tentent de les prendre par force ou par traitrise. Ils s'approprient leurs marchandises et font prisonniers les passagers dont ils font de nombreux esclaves; et ils en font autant avec les Portugais et les Espagnols qui passent par-là dont ils peuvent se saisir ${ }^{4}$. Ils ont pour armes naturelles la lance et le bouclier; ils utilisent maintenant quelques pièces d'artillerie et d'arquebuserie, et ce sont les esclaves étrangers qui les servent, car ils ont eux-mêmes peu de goût pour cela et ils s'en servent plus pour effrayer qu'effectivement.

Ils ont des rites abominables qui donnent la nausée au seul fait de les décrire ${ }^{5}$. Il y a en ce royaume de nombreux Mahométans et le roi désire et se fait un plaisir que l'on dise et enseigne la secte de Mahomet à tous ses sujets et, à cet effet, on y trouve de nombreuses mosquées ${ }^{6}$. Il y a aussi de nombreux autres temples de gentils? Le roi est un grand ennemi des Chrétiens et de notre Sainte Loi, car il dit qu'il est trop incommode de la pratiquer; il n'a pas consenti à la prédication du Saint Evangile ${ }^{8}$.

(1) Les Cam sont renommés pour la construction de leurs charrettes d'un type très particulier puisque leurs deux roues sont montées sur des moyeux indépendants (voir par exemple Leuba 1923 : 120-121).

(2) Cette interdiction est bien entendu liée à l'hindouisme pratiqué par les Cam. Le Sejarah Melayu, dans son chapitre consacré au Campā, notait aussi cette particularité (Marrison 1951 : 92, 95).

(3) Il sufft de se reporter à la statuaire cam pour avoir confirmation de cette afmrmation (Boisselier 1963) ou, pour une période plus récente, à Leuba 1923 : 83.

(4) Plusieurs des auteurs de cette relation en ont fait la malheureuse expérience (supra, p. 255). Les Hollandais dont les Cam pourront se saisir seront eux aussi à plusieurs reprises réduits à l'esclavage (Buch, $1936: 173$ ).

(5) Ces rites abominables *, outre la sali des veuves, consistent surtout en sacrifices humains pendant lesquels le flel est récolté pour être amené au souverain. Ils sont décrits avec force détails dans de nombreux documents ibériques de l'époque et en particulier dans la relation publiée par Boxer $(1970: 39,42)$. A la fin du xir' siècle, Zhou Da Guan fait une description de la récolte du flel au Cambodge pour le compte du roi du Campā, qui ressemble de très près à cette dernière, à ceci près que le document du xvie siècle amrme que seul le fiel des sujets cam est accepté (cf. Pelliot $1902: 173$ et la longue note qu'il y consacre aux croyances attachées au fiel dans le sud-est asiatique).

(6) Sur l'Islam et son introduction au Campã, voir Manguin 1979.

(7) C'est-à-dire des temples hindouĩstes.

(8) Ce n'est qu'au xvı ${ }^{\text {e }}$ siècle, semble-t-il, que les missionnaires ont pénétré au Campā, et encore l'ont-ils fait à la suite de la poussée viêtnamienne vers le Sud. Il semble que beaucoup de Viêtnamiens chrétiens aient profté de cette migration pour échapper aux persécutions (voir par exemple la lettre annuelle de la Compagnie de Jésus dont un paragraphe est consacré 
Ce site est vraiment la place la plus importante de toutes celles situées sur les côtes de cette mer [de Chine], pour les susdites raisons. Eu égard à sa disposition, il me semble en Dieu et en conscience que l'on peut la prendre avec 150 Espagnols et 500 Indiens et la maintenir ainsi, et maîtriser toute cette mer et ceux qui y passent. Car en dépit du fait qu'il s'agit d'un petit nombre d'Espagnols en comparaison du nombre d'habitants, il faut remarquer que l'on ne combat qu'avec le roi et avec ceux de la ville, et que celle-ci est bien disposée pour être prise, car elle est en bordure de la mer et qu'en outre son peuple est faible et sans courage, ses armes de mauvaise qualité et ils sont malhabiles lorsqu'ils s'en servent et très désordonnés lors des combats. Ces gens sont en outre tyrannisés par les rois : ils ne peuvent posséder objet de valeur ou de prix dont ne s'emparent aussitôt le roi ou ses grands, de sorte que ce sont plutôt des esclaves que des vassaux. La ville possède dix pièces d'artillerie à culasse, démontées et pleines de sable, pesant jusqu'à 25 quintaux. Elle possède aussi 500 barses réparties entre les maisons du roi et de ses grands, dans des magasins, qui sont utilisées lorsqu'ils sortent se battre en mer ${ }^{1}$ et ils se servent de même de leurs arquebuses, dont ils possèdent près d'un millier, mal ajustées et avec une poudre de très mauvaise qualité. Si j'affirme cela c'est que je l'ai vu. Sa Majesté pourra faire usage de toutes ces armes, de même que ceux qui s'empareront de la ville, de façon à la défendre.

Nous tous qui apposons ci-dessous notre signature, affirmons et certifions que cette relation est exacte et véridique, et nous en avons connaissance car certains d'entre nous l'ont vu, d'autres l'ont appris de sources très sûres, et parce que tout ceci est de notoriété publique et que nous en avons souvent entendu parler par les nombreuses personnes qui l'ont vu et par de nombreux autochtones de ces mêmes royaumes.

Fait à Manille, le 7 décembre 1595. De visu : Blas Ruiz de Hernan Gonçales. Par ouï-dire, de la relation que m'en ont fait des Portugais de Macau, disant combien ce royaume était important pour Sa Majesté : le capitaine Diego de Chaves Cañizares. De récits explicites que l'on m'en a fait au Cambodge, son voisin, où j'ai habité neuf années et où résident de nombreux Cam : Diego Beloso. De visu : Gregorio de Bargas Machuca. De visu : Francisco de Sagredo. De récits faits à terre et pour l'avoir vu de la mer: Pantaleon Carnero.

à la * mission du Campā en 1686 (rêférences supra, p. 256, n. 2) ; les Pères des Missions Étrangères s'y trouvaient aussi dans la deuxième moitié du xvı́ siècle et se consacraient essentiellement aux Viêtnamiens, bien qu'ils semblent avoir tenté de convertir des Cam (l'un d'entre eux reçoit en 1685 un Coran ; Launay 1923-25, I : 243-244, 352-353 ; II : 132-134, 193-195. Voir aussi les documents des Archives de la Société des Missions Étrangères cités in Manguin 1979 : 271-272, 275).

(1) L'artillerie lourde, destinée à la défense ou au siège des fortifications, n'a jamais été le point fort des armées sud-est asiatiques qui ont le plus souvent fait appel, pour la servir, à des artilleurs turcs ou européens. Par contre, les pièces légères - barses (versos), pierriers, fauconneaux, etc. - étaient très communes lors des combats navals ou sur les estacades défendant les villes légèrement fortifeees de la frange maritime du sud-est asiatique. Très maniables car montées sur pivot, parfois assez petites pour être portées par un seul homme, elles étaient utilisées contre les combattants (et tiraient de ce fait souvent à mitraille). On voit ici que le Campā n'échappait pas à cette règle. Sur l'usage de l'artillerie dans la région, voir Boxer 1965, Shariffudin 1969, Manguin 1976 b et les références qui sont données dans ces trois études. 


\section{L'extension territoriale el la capitale du Campā au XVIe siècle}

Les indications d'ordre géographique données dans la "Relation" sont rien moins que précises, en dépit des témoignages de visu de certains des cosignataires. Trois chiffres sont donnés dans son premier paragraphe qui se révèlent être tous les trois erronés. Si la capitale cam avait été située par $10^{\circ}$ de latitude nord, elle se serait trouvée en plein dans les bouches du Mékong, ce qui est évidemment absurde (ne serait-ce que parce qu'on est là en dehors du territoire cam). Par ailleurs, de sa frontière méridionale avec le royaume khmer à sa frontière septentrionale avec le Đại-việt (la "Cochinchine"), la côte cam se serait étendue sur 100 lieues ( 320 milles nautiques; $590 \mathrm{~km}$ ). Or ces frontières sont connues avec assez de précision pour que l'on puisse déterminer la distance qui les sépare et qui est, tout au plus, de 240 milles nautiques (440 km).

Au nord, après la prise de Vijaya en 1471, le souverain Lê établit la frontière du Đại-việt au cap Varella, le Thạch-bi sơn ou Núi Đá Bia (la "montagne de la stèle ") : c'est précisément à la borne frontière que fait allusion ce toponyme ${ }^{1}$. Ce n'est qu'en 1653 , après une nouvelle incursion punitive contre les Cam, que cette frontière sera déplacée vers le sud².

(1) Hông-đức bản đồ (éd. Bừu-câm et alia), Sài-gòn $1962: 49,158$. Lê Quí Đôn, Phủ biên lạp lucc (éd. en quôc-ngur) Hà-nội $1964: 125$.

(2) Ici encore les sources divergent. On semble généralement accepter, à la suite de Lê Qui Đòn, le fait que ce fleuve de Phan-rang ait servi de frontière, la rive sud restant aux mains des Cam (Aurousseau 1920:87 n. 1; Maspero 1928:241 n. 2). Et il est certain que le Đại-nam liệt-truyện (chinh biên, sơ $t a ̣ ̂ p, q .33: 21$ b) parle sans équivoque de zones d'influence réparties de part et d'autre du fleuve. Mais la frontière elle-même pourrait avoir été matérialisée plus au nord. C'est ce que laissent penser un certain nombre d'autres sources tant européennes que viêtnamiennes. * La ville de Nha-rou qui est le commencement du Royaume de Cochinchine ", dit une relation de 1665 (Relation des missions des Evesques Francois (...), Paris 1674 : 107). Ce " Nha-rou "n'est autre que le Nha-du du Hông-đức bân đô, immédiatement au nord de Nha-trang (Manguin $1972: 146 \mathrm{n}$. 1). La frontière doit donc être située à son sud. Le journal de route d'un missionnaire des années 1680 donne trois jours de Phan-ri à Phan-rang et deux journées et demie de Phan-rang à la frontière de Cochinchine, ce qui exclut d'emblée la rive nord du fleuve de Phan-rang et nous mène plus au nord dans les environs de Cam-ranh (Launay 1923-25, I : 353). Ce sont enfin deux des cartes du Hông-đức bán ấ (éd. 1962 : 101, 163) et des autres ouvrages géographiques viêtnamiens de même catégorie (Kiên khôn nhât lãm : 29a, 62a, Ms. A 414 de l'ex-fonds de l'EFEO à Hà-nội ; Nam-vięt bản đồ : 52a, 89a, Ms. A 1603 de l'EFEO), qui situent une stèle frontière entre les deux pays, en arrière de la baie de Camranh. Mais le texte joint à l'une de ces deux cartes (intitulée Thién-nam tứ-chi lọ $d \hat{o}$ thur) lie cette borne aux événements de 1471 relatifs à l'établissement de la frontière au Cap Varella : l'erreur vient-elle alors de ce seul texte -- dont l'auteur aurait pu confondre cette stèle avec celle de 1471 , toutes deux portant sur les cartes la légende thach-bi-, on bien les cartes elles-mêmes sont-elles toutes pareillement erronées? Les remarques des missionnaires français, citées plus haut, donnent à penser que ces cartes traduisent bien en fait la réalité en situant dans les environs de Cam-ranh cette frontière (cela résoudrait la question que se pose à ce propos l'éditeur du Hông-đức bån $a \hat{o}, 1962$ : xiii, xxvi). 
En ce qui concerne la frontière entre les royaumes cam et khmer, les sources disponibles ne sont pas unanimes. Un routier portugais de la fin $\mathrm{du} \mathrm{XvI}^{\mathrm{e}}$ siècle indique sans équivoque possible que les confins des deux royaumes sont marqués par les hautes montagnes en face desquelles, à quelque 10 milles au large, est située la "Roche de Matheus de Brito "1, ce qui revient à placer cette frontière, sur la côte, par environ $10^{\circ} 35^{\prime}$ de latitude nord. D'Après de Mannevillette, dans son Neptune Oriental, la situe de même par $10^{\circ} 30^{\prime 2}$. C'est cette solution que j'avais adoptée dans mon commentaire à ce routier, me fiant alors à la grande précision dont fait, en général, preuve cette catégorie de sources ${ }^{3}$. Mais il semble bien en fait, à en juger par la majorité des autres témoignages, qu'il faille placer cette frontière légèrement plus au sud, à la hauteur du cap Saint-Jacques (Vũng Tàu) .

En 1637, les Hollandais situent cette frontière "à proximité " de ce qu'ils nomment la "rivière japonaise ${ }^{5}$ ". D'après la carte hollandaise jointe par Buch à son article, comme d'après le Neptune Oriental et ses cartes, cette "rivière japonaise " est la branche septentrionale du delta du Mékong, le Sông Mỹ-tho, qu'empruntaient les navires ${ }^{5}$. Mais en situant la frontière aussi loin vers le sud on placerait le site de Sài-gòn, dont on sait qu'il était en territoire khmer, en pays cam. Il faut donc selon toute vraisemblance étendre la "proximité " de cette rivière jusqu'au cap Saint-Jacques qui fait face à son embouchure, légèrement plus au nord. Christoval de Jaque, au début du Xvil ${ }^{e}$ siècle, cite "un port situé précisément à la frontière des deux Etats, auquel Vincent Fernandes, notre pilote, donne le nom de Cinq Plaies, parce qu'on y voit cinq montagnes élevées?". Ces "Cinq Plaies" (les stigmates du Christ) sont la traduction du toponyme portugais "Cinco Chagas ", dont la déformation a donné en dernier ressort le Saint-Jacques des Français ${ }^{8}$. Le port est donc celui de Vũng Tàu, à l'abri du cap du même nom. Or, sur la carte du Giáp-ngo binh nam dô (de la fin du XviI ${ }^{\mathrm{e}}$ siècle $^{9}$ ), la frontière entre Campā et Cambodge (Chiêm-thành giáp Cao-miên giới)

(1) Banc de Britto " sur les cartes du Service Hydrographique de la Marine, par 10³0' de lat. nord.

(2) Op. cil. : 155.

(3) Manguin 1972: $81 \mathrm{n} .6$ et carte 3 . Une façon de réconcilier ce témoignage avec ceux qui suivent serait de comprendre que ces montagnes marquent les confins des deux royaumes dans l'arrière-pays, sans pour autant indiquer leur frontière jusqu'à la cóte : le Campā serait ainsi constitué d'une étroite bande littorale.

(4) Je me range donc ici aux arguments de feu $M^{11}$ Martine Piat qui m'avait personnellement communiqué en 1970 certaines des références à des textes faisant mention de cette frontière utilisés ici. C'est malheureusement à titre posthume que je dois lui exprimer ici mes remerciements.

(5) Buch 1936(-37) : 202.

(6) Sur les bouches du Mékong et leur toponymie aux xvı et xvire siècles, cf. Manguin 1972 : 95 n. 1.

(7) C. de Jaque de los Rios Mancaned, Voyages anx Indes orientales et occidentales (...), in Ternaux-Compans 1940, $1: 262$.

(8) Manguin $1972: 93$ n. 5.

(9) Hông-đức bớn đồ $1962: 164-165$. 
est indiquée au sud de Xích-lam et au nord de l'embouchure de Mỹ-tho, à l'aplomb d'un estuaire de Tắc-kê que je n'ai pas pu identifier mais qui, en toute logique, se trouve dans les environs du cap Saint-Jacques ${ }^{2}$. Une dernière source, plus tardive puisqu'elle date de 1725 , place cette frontière sur la côte "face à Baria ", ce qui nous mène dans les environs du cap Saint-Jacques ${ }^{3}$.

Par ailleurs, l'erreur de la "Relation" concernant la latitude de la capitale cam est malheureuse, car elle nous enlève la seule information précise que nous aurions eue quant à sa situation au xvi ${ }^{e}$ siècle $^{4}$. Il faut remarquer ici que toutes les sources européennes qui indiquent sans équivoque possible que la capitale cam est à Phan-ri ${ }^{5}$ datent de la deuxième moitié $d u$ XVII $^{\mathrm{e}}$ siècle, après l'annexion en 1653 par le Đại-việt de la partie septentrionale du territoire cam ${ }^{6}$.

Gabriel Quiroga de San Antonio (1604) mentionne le "Royaume de Champa (...) situé par $11^{\circ}$ de latitude ", ce qui en soi est absurde et laisse penser que c'est le parallèle de la capitale auquel il est fait allusion?. Mais si $11^{\circ}$ nous mènent plus près de Phan-ri $\left(11^{\circ} 10^{\prime}\right)$ que de Phan-rang $\left(1^{\circ} 33^{\prime}\right)$, l'approximation est telle que l'on ne peut en tirer de conclusion. En 1607 , le roi " tenait sa cour au nord du cap situé par $11^{\circ}$ de latitude ", nous informe un récit hollandais ${ }^{8}$. Ce $11^{\mathrm{e}}$ parallèle passe à mi-hauteur entre deux caps, le Mũi La-gàn par $11^{\circ} 10^{\prime}$, c'est-à-dire la latitude de Phan-ri, et le Mũi Vị-nê par $10^{\circ} 55^{\prime}$. Au premier abord, ce témoignage semblerait indiquer Phan-ri $\left(11^{\circ} 10^{\prime}\right)^{9}$. Mais ces deux caps sont peu

(1) Xich-lam doit être situé par $10^{\circ} 28^{\prime}$ lat. nord, à une trentaine de kilomètres au nord-est du cap Saint-Jacques, près du village de Lộc-an (communication personnelle de G. Moussay). Voir aussi à ce propos la carte de la province de Biên-hoà du Đại-nam toàn đô (Ms. A 2559 de l'ex-fonds de l'EFEO à Hà-nội) et les indications du Đại-nam nhất thông chí (éd. Hà-nội, $1971, \mathrm{~V}: 58$ ). En me fondant sur les indications erronées d'un routier portugais et n'ayant pas vu la correspondance Sidrão $=X$ ich-lam, j'avais proposé par erreur la baie de Phan-thiêt (Manguin $1972: 88 \mathrm{n} .3$ et 168).

(2) Hông-đức bản đô 1962: 166-167. L'éditeur a transcrit et traduit cette indication de frontière comme faisant partie du texte accompagnant la carte. Mais, comme on peut en juger par l'examen d'autres copies plus claires de cette carte, il s'agit bien d'une des légendes de la carte elle-même, et il faut donc tenir compte de sa position géographique (voir par exemple la Nam-việt bán-đô, fol. 91 ; ms. A 1603 de l'ex-fonds de l'EFEO à Hà-nội).

(3) Lettre du P. José de la Concepción OFM, publiée in Perez 1922 : 301.

(4) Toutes les copies de ce texte comportent cette erreur et elle se trouvait donc dans l'original.

(5) Le Đại-nam nhât-thông chi (q. 12, éd. Sài-gòn $1965: 41$ ), rédigé en plein xıxe siècle, dans son chapitre sur les vestiges de la province de Binh-thuận, place une ancienne capitale cam au village de Vĩnh-an, huyę̆n de Hoà-đa, au sud-ouest de la capitale provinciale de Xuân-an, mais sans mieux préciser la date de l'occupation de ce site. Il semble qu'il s'agisse du même site que celui de Tịnh-mỹ (district de Phan-lý Cham) qui était encore il y a quelques années la résidence des descendants de la famille royale cam (Durand 1905 c : 382-386; Pó Dharma $1978: 119)$.

(6) Voir par exemple la Relation des Missions de Cochinchine de 1685 in Launay 1923-25, I : 352, ou le témoignage de 1720 cité dans Le Neptune Oriental (op. cit. : 148 sq.).

(7) Cabaton $1914: 122$.

(8) Historische Verhael van de treffelijcke Reyse (...) door Cornelis Matelief de Ionge, inden jaren 1605, 1606, 1607, 1608, dans Begin ende Voortgang (...), s.1., 1646 : 499-502.

(9) C'est l'hypothèse que j'avais retenue lors d'un premier examen de cette information (Manguin $1979: 270$ ). 
notoires et c'est bien plutôt à l'imposant promontoire du cap Padaran (Mũi Dĩnh, $11^{\circ} 22^{\prime}$ ) que l'on est amené à penser ici : c'est sur ce cap, à l'aplomb duquel les navires devaient passer et changer de route que les textes disponibles insistent toujours, comme le fait d'ailleurs la "Relation" présentée ici. Si le roi tient sa cour au nord de ce cap, c'est donc qu'elle se trouve dans les environs de Phan-rang. Un routier portugais antérieur à 1595, enfin, nomme Phan-rang "le Port de Campā", ce qui pourrait impliquer qu'il s'agit de la capitale du pays".

Les témoignages européens restant peu concluants, il faut sc tourner vers d'autres sources. Si, comme on va le voir, celles-ci ne sont pas beaucoup plus précises, du moins semblent-elles bien faire pencher encore la balance en faveur d'une localisation de cette capitale jusqu'en 1653 dans la plaine de Phan-rang'. D'après J. Boisselier, qui se fonde sur l'évolution stylistique, "si le temple de Po Romé semble bien être la construction en matériaux durables la plus tardive du Champa, il est douteux qu'il puisse être d'une date plus basse que le cours du XVI ${ }^{\mathrm{e}}$ siècle ". De même le mukhalinga de Po Klaung Garai, qui appartient au style de Po Romé, devrait-il être daté de la fin du Xvi ou du début du XVII ${ }^{e}$ siècle $^{3}$. Il réfute ainsi la thèse d'E. Durand ${ }^{4}$ qui, se fondant sur la lecture très hypothétique d'inscriptions et de graffiti trouvés in situ, associait ce temple au règne du Po Romé (Pŏ ramō) des Chroniques cam; celui-ci aurait été au pouvoir, si l'on accepte les computs d'Aymonier et de Pŏ Dharma, de 1626 à 1651 . Que l'on retienne l'une et l'autre de ces thèses, on reste dans la période précédant l'attaque viêtnamienne de 1653 et le probable transfert de la capitale à Phan-ri. On peut donc penser que la fondation de ce temple - le dernier édifié en matériaux durables, rappelons-le - ait été lié à la proximité de la capitale cam du moment. Or G. Moussay, se fondant sur des reconnaissances personnelles, pense avoir reconnu le site de la capitale dans les environs de ce temple, près du village actuel de Phú-bôn ${ }^{5}$. Par ailleurs, les traditions orales recueillies par $H$. Parmentier placent une capitale à $6 \mathrm{~km}$ à vol d'oiseau vers le sud de l'actuelle Phan-rang, près du village de Chung-mỹ, un site qui diffère donc de celui repéré par G. Moussay. Une autre tradition orale recueillie par Pŏ Dharma" veut que le lac dit "de Phan rang ", à Chung-mỹ, ait été situé à proximité du palais du roi. En tout état de cause, entre ces deux sites éloignés d'environ $6 \mathrm{~km}$, on trouve un grand nombre de vestiges divers dont, semble-t-il, des traces de murailles?. Tous ces éléments portent donc bien à croire que la capitale cam a été située dans cette région dans la première moitié du Xvil ${ }^{\mathrm{e}}$ siècle, avant d'être transférée plus au sud après les combats de 1653 .

(1) Manguin $1972: 82$.

(2) Les arguments catégoriques d'E. Durand (1905 a, b, c) selon lesquels la capitale cam aurait été située à Phan-ri dès 1579 sont rien moins que convaincants : faisant $\mathrm{f}$ des règles de phonétique, maniant allègrement l'anachronisme, il obscurcit le problème mieux qu'il ne le résoud.

(3) Boisselier $1963: 386-387$.

(4) Durand 1903 : 601 sq.

(5) Communication personnelle.

(6) Parmentier 1909 : 73-74; Pó Dharma 1978 : 118.

(7) Communication personnelle de Pó Dharma. 


\section{APPENDICE B}

\section{Les manuscrits de la "Relaçion de las cossas de Chanpa"}

Il existe de nombreux manuscrits de la "Relation" qui est ici présentée. La transcription de son texte a été établie à partir de la copie contemporaine qui est conservée à l'Archivo General de Indias de Séville (collection Patronato, legajo 25, ramo $3^{\circ}$, doc. 58, fol. $7 v^{\circ}-9 \mathrm{r}^{\circ}$ ), que l'on a complétée par une autre copie --- légèrement plus tardive -. conservée dans ces mêmes archives dans la collection Filipinas (Legajo 19, où elle accompagne une lettre originale du gouverneur Luiz Pérez Dasmariñas au roi Philippe II, datée de Manille, le 30 juin 1603). Toujours en Espagne, le Museo Naval de Madrid possède dans sa collection Navarrete (vol. 18, fol. $302 \mathrm{r}^{0}-307 \mathrm{r}^{\circ}$ ) une copie du premier de ces documents, effectuée au Xviı ${ }^{\mathrm{e}}$ siècle (son texte est assez corrompu, le copiste travaillant pour Navarrete ayant pris la liberté de moderniser en partie l'orthographe et de sauter quelques mots). La bibliothèque de l'École Française d'Extrême-Orient à Paris possède enfin un volume manuscrit de copies effectuées à Séville en 1908 pour Antoine Cabaton lors de sa mission dans la Péninsule Ibérique, qui inclut les deux versions précitées de ce texte (Ms. Européen 125, fol. $\left.84 v^{0}-87 v^{o}, 160 \mathrm{r}^{\circ}-163 \mathrm{r}^{\circ}\right)^{1}$. On connaît enfin l'existence, mais non le lieu actuel de conservation, d'une dernière copie de cette "Relation" effectuée, semble-t-il, à Manille sur les originaux en la possession du gouverneur. Ce manuscrit a été mis en vente à Londres chez Maggs Bros. en 1929 au sein d'un lot d'autres textes rédigés à Manille vers 1595-1600 et portant sur l'Asie du Sud-Est². Ce même lot de manuscrits philippins s'est retrouvé en vente à Paris en 1955 chez Adrien Maisonneuve ${ }^{3}$ et le texte qui nous intéresse ici a été à nouveau mis en vente en 1967 chez H. P. Kraus à New York.

(1) Cr. le rapport de mission d'A. Cabaton (1910). Mais il n'y est pas fait nommément mention de ce document.

(2) Catalogue de Maggs Bros., n० 515, 1929, Bibliotheca Asiatica, part III ; 21-22, où l'on trouvera un bon résumé en anglais du document publié ici (il y porte le $n^{\circ} 5$ du lot mis en vente).

(3) Catalogue $n^{\circ} 51$, sous le numéro 8944.

(4) Sur l'historique de ce lot de manuscrits philippins, on se reportera aux annotations de C. R. Boxer (Groslier et Boxer 1958: 178 et $1970: 35-36$ et $n$. 3) ; cet auteur n'a eu connaissance du manuscrit publié ici qu'à travers le résumé du catalogue de Maggs Bros. 


\section{APPENDICE G}

\section{Texte espagnol}

[7vo] Relaçion de las cossas de Chanpa

En cumplimiento de lo que V.Sa. me manda de que haga relaçion del sitio que tiene el reino de Chanpa y de las cossas que ay en el, digo :

Que la çiudad principal del dicho reyno adonde el rei asiste esta en la rivera de la mar, en una punta de la tierra firme que sale mui fuera a la mar. Esta en altura de 10 grados. La costa que desta çiudad y punta corre a la vanda del norte se estiende 40 leguas hasta el reino de Cochinchina, con quien confina por esta parte. La costa que desde la dicha çiudad y punta por la otra parte corre al lueste se estiende 60 leguas de costa hasta el reino de Camboja con quien confina.

Con la dicha punta y çiudad hazen estrecho de seis leguas unos bajos que vienen corriendo la costa de la vanda del norte 200 leguas, apartados de tierra firme $\mathbf{4 0}$ leguas, y hazen el remate en la dicha punta y ciudad de Chanpa, y su principio es frontero de la ysla de Enao que esta en la costa de la China. El que tuviere este sitio y punta sera señor de toda esta mar, porque desde este dicho estrecho hazia la parte del norte estan los reinos de Cochinchina y la gran China, y los de Japon, y a la parte del sur del dicho estrecho estan los reinos de Canboja y Siam, Patan, Paan, Joor, Andregui, Sunda, Samatra, Solor, Timor, Yslas de Vanda, y la gran Java y Burney y Malaca y todas las partes de la Yndia Oriental. [8 $\mathrm{r}^{\circ}$ ] Todos los dichos reinos de la parte del sur del dicho estrecho e punta tienen trato y comerçio con los de la vanda del norte, y los otros con ellos communican en si de una parte a otra grandes y gruesas mercadurias, muy ricos tratos, y para su navegaçion es passo forssoso el dicho estrecho de yda y vuelta de todos en sus tiempos, porque no tienen otro camino y les es fuerça passar pegados a la tierra firme y punta, por que es costa sondable y linpia, y por huir de los bajos y de unas grandes corrientes que tienen. Tanbien pasan por el dicho estrecho las naos de los Portugueses que van de la Yndia a China y Japon, y por aqui mismo passan a la vuelta de sus viajes porque, como digo, no hay otro camino y ser les a ellos grande comodidad para sus viajes y seguridad el ser aquel sitio poseido por $\mathrm{Su} \mathrm{M}^{\mathrm{d}}$., porque esta cassi en la mitad del camino de Malaca a China y podran tener alli

(1) Cette transcription est fondée sur la version de l'Archivo General de Indias de Séville (Patronato, legajo 25). Les abréviations en ont été développées, les mots séparés, la ponctuation introduite et les majuscules rétablies selon l'usage moderne. L'orthographe du texte a été conservée, à l'exception de double $r$ à l'initiale. Entre crochets ont été ajoutés quelques mots ou passages qui apparaissent dans la seule copie de 1603 (Filipinas, legajo 19), mais qui devaient figurer sur l'original. 
refresco y hazer ynvernada, que por temor de los Chanpanes no lo hazen y se ponen muchas vezes a mucho riesgo, assi a yda como a vuelta, por yr algunas vezes tarde a sus viajes y por ynvierno.

Es tierra [llana y enjuta y] muy apaçible, razonablemente poblada; tiene muy buen cielo y tenple sano. Produze muchas mercadurias como es ropa de algodon y de seda, çera y hierro, y tiene muchos montes de ebano, elefantes de que tiene mucho marfyl, e abadas e muchas cossas dellas. Hay un palo que llaman calanba que tiene muchas virtudes, prieto y olorosso y de mucho valor, mucha madera y muy buena para haçer naos, y mucho aparejo para hazerlas, porque todos los naturales son carpinteros $\mathrm{y}$ herreros $\mathrm{y}$ por aver como digo hierro en la tierra. Tiene muchos bastimentos como el arroz, vino, vacas, gallinas, venados, puercos, cabras, bufanos, [liebres, perdiçes, conejos, tortolas, pabos reales] y mucho pescado; ay muchos caballos de que se sirven, y de elefantes que hay muchos mansos. Tienen muchas carretas de vacas y bufanos. No pueden los naturales matar ni comer vaca, que es lei entre ellos, y esta es la caussa de que ay muchas.

La gente de este reino esta vestida de ropa de algodon y de seda. Traen el cavello largo y atado en çima de la caveça. Es gente muy devil y muy pusilanima, enemigos de armas, viçiosos, amigos de fiestas y pasatienpos; y lo que les caussa este viçio y descuido es el sitio tan fuerte $\mathrm{y}$ passo tan forsoso para todos los demas reinos $\mathrm{y}$ tener sus contrataçiones como $\left[8 \mathrm{v}^{\circ}\right]$ hemos dicho, y porque salen a rovar a todos los navios flacos y descuydados que por alli passan, y los procuran tomar por fuerça o por traiçion, $y$ se aprovechan de sus haçiendas y les cautivan las personas, de que tienen muchos esclavos, y lo proprio hazen de Españoles y Portugueses que pueden aver de los que por alli passan. Tienen por armas naturales lança y rodela, usan agora alguna artilleria y arcabuzeria, y esta exerçitan los estrangeros esclavos, porque ellos no tienen animo para ello, que mas les sirve para espanto que para hazer efecto.

Tienen ritos abominables que provocan asco escrevillos. Ay en el reino muchos Maometanos y quiere el rey y gusta de que a toda la gente de su reino se les diga y enseñe la seta de Maoma y para este efecto ay muchas mesquitas aunque ay otros muchos tenplos de gentiles. Es muy enemigo de Cristianos y de nuestra Santa Ley, porque dize que es de mucho travajo el guardarla. No ha consentido predicar el Santo Evangelio.

En efecto este sitio que esta dicho es la plaça mas importante de todas quantas ay en la tierra firme desta mar, por las caussas que arriba estan dichas, y sigun su dispossiçion me pareçe en Dios y en mi conçencia que se puede tomar con 150 Españoles y 500 Yndios y sustentarla y señorear toda aquella mar, y las gentes que por alli pasan, porque aunque es poco numero de Españoles para la mucha gente de la tierra, deve se advertir que solo se pelea con el rei, y con la gente de la çiudad, y que tiene grande aparejo para tomarla por ser a la rivera de la mar, y demas desto ser la gente flaca y sin animo, y malas armas, no diestros en ellas y sin orden ninguna en el pelear. [ $Y$ es gente tiraniçada de sus reyes, que no pueden tener cosa de valor ni preçio que luego se la 
quitan el rey o sus grandes de manera que mas le son esclavos que vassalos.] Tiene la çiudad hasta diez pieças de artilleria de culata, desencabalgadas y llenas de arena, de a 25 quintales; hasta 500 versos repartidos en cassa del rey y de los principales, metidos en almazenes, para quando sale a pelear a la mar, y para el mismo efecto se sirve de sus alcavuçes que tendra hasta mil, mal adereçados y con mui mala polvora. Y esto digo por que lo e visto. De todas estas armas se podra aprovechar Su Md. y la gente que tomare la çiudad para defensa della propria.

Todos los que aqui firmamos nuestros nombres, dezimos y certificamos que esta relaçion es çierta y verdadera, y lo savemos porque lo avemos visto, $\mathrm{y}$ algunos de notiçias muy claras y de publisidad que ay dello, y que lo emos oydo muchas vezes [9ro] a muchos que lo han visto y a muchos naturales de los mismos reinos.

Fecho en Manila a 7 de diziembre de 1595. De vista : Blas Ruiz de Hernan Gonçales. De notiçia, por relaçion que me hizieron en Macau Portugueses, diziendo quanto ymportava a $\mathrm{Su} \mathrm{Md}^{\mathrm{d}}$. aquel reino : el Capitan Diego de Chaves Cañizares. De notiçia clara que dello tuve en el reino de Canboja su convezino, donde asisti nueve anos y ay muchos Chanpanes moradores : Diego Beloso. De vista : Gregorio de Bargas Machuca. De vista : Francisco de Sagredo. De noticia de tierra y vista de mar : Pantaleon Carnero. 


\section{BIBLIOGRAPHIE}

Aurousseau, L., 1920. - "Compte rendu de Ch. B. Maybon, Histoire moderne du pays d'Annam ", BEFEO, XX/4 : 73-120.

Boisselier, J., 1963. - L L statuaire du Champa (PEFEO LIV), Paris.

Boxer, C. R., 1950. -- "A late sixteenth-century Manila Manuscript", Jl. of the Royal Asiatic Society, April : 37-49.

- 1965. - "Asian Potentates and European Artillery in the 16th-18th centuries ", Jl. of the Malaysian Branch of the Royal Asiatic Society, XXXVII/2 : 156-172.

-- 1969. - " Portuguese and Spanish Projects for the Conquest of Southeast Asia ", Jl. of Asian History, III/2 : 118-136.

- 1970. - "A Spanish Description of the Chams in 1595 ", in : Readings on Asian Topics (Scandinavian Institute of Asian Studies, Monograph no 1), Lund : 35-44.

Buch, W. J. M., 1936-1937. -- "La Compagnie des Indes Néerlandaises et l'Indochine », BEFEO, XXXVI : 97-196; XXXVII : 121-237.

Cabaton, A., 1910. -- "Missions en Espagne et en Portugal 》, Bulletin de Géographie historique et descriptive, $\mathrm{n}^{\circ}$ 1-2 : 15-36.

- 1914-1916. ... "Le Mémorial de Pedro Sevil à Philippe III sur la conquête de l'Indochine (1603)", Bulletin de la Commission archéologique de l'Indochine : 1-102.

- 1914. - Brève el véridique relation des événements du Cambodge par Gabriel Quiroga de San Antonio, Paris.

Contesão, A., 1944. - The Suma Oriental of Tomé Pires (Hakluyt Society), London, 2 vol.

Dames, W. L., 1918. - The Book of Duarle Barbosa (Hakluyt Society), London, 2 vol.

Durand, E. M., 1903. -... "Le temple de Po Romé à Phan-rang", BEFEO, III : 597-603.

- 1905a. - "Parik-Pinān et le clan de l'Aréquier », BEFEO, V : 368-373.

-.-. 1905 b. - "La Chronique royale ", $B E F E O, \quad \mathrm{~V}: 377-382$.

- 1905 c. - " Bal Canar ", BEFEO, V : 382-386.

Ferrand, G., 1913-1914. … Relations de voyages el textes géographiques arabes, persans et turks relatifs à l'Extrême-Orient. Paris, 2 vol.

Grimm, T., 1961. - "Thailand in the Light of Official Chinese Historiography : a Chapter in the "History of the Ming Dynasty", Jl. of the Siam Society, XLIX/1 : 1-20. 
Groslier, G. et G. R. Boxer, 1958. - Angkor et le Cambodge au $X V I \mathrm{e}$ siècle d'après les sources portugaises, Paris.

Launay, A., 1923-1925. ... Histoire de la Mission de Cochinchine. Documents historiques. Paris, 3 vol.

Leuba, J., 1923. - Les Chams et leur art, Paris et Bruxelles.

Manguin, P.-Y., 1972. - Les Portugais sur les côtes du Viêt-nam et du Campä (PEFEO LXXXI), Paris.

1976 a. -... "La traversée de la Mer de Chine méridionale, des Détroits à Canton, jusqu'au xvile siècle ", in : Acles du $X X I X^{\mathrm{e}}$ Congrès International des Orientalistes (Paris 1973). Section Asie du sud-est continentale, Paris, vol. II : 110-115.

- 1976 b. - "L'artillerie légère nousantarienne ", Arts Asiatiques, XXXII : 233-268.

- 1979. - "L'introduction de l'Islam au Campā", BEFEO, LXVI : 255-287.

Marrison, G. E., 1951. - "The Chams of Malacca ", Jl. of the Malaysian Branch of the Royal Asiatic Society, XXIV/1 : 90-98.

Maspero, G., 1928. - Le Royaume de Champa, Paris.

Parmentier, H., 1909. - Inventaire descriptif des monuments cams de l'Annam, vol. I (PEFEO XI), Paris.

Pelliot, P., 1902. "- Mémoire sur les coutumes du Cambodge par Tcheou-Ta-Kouan ", BEFEO, II : 123-177.

Perez, L., 1922. - "Los Españoles en el Imperio de Annam. Missiones en Cochinchina, Chiampa y Cambodja ", Archivo Ibero-Americano, XVII : 293-340.

Po Dharma, 1978. - Chroniques du Panduranga. Introduction, textes et traductions. Diplôme de l'École Pratique des Hautes Études (IVe section), Paris (inédit).

Shariffuddin, P. N., 1969. - "Brunei Cannon", Brunei Museum Journal, I/1 : 72-93.

Sion, J., 1929. - Asie des Moussons, 2 e partie, Paris.

Stern, R., 1947. - "Le Lin-yi, sa localisation, sa contribution à la formation du Champa et ses liens avec la Chine", Han Hiue, II/1-3.

TernauX-Compans, H., 1840.--Archives des voyages (...), Paris, vol. I.

Yule, H. et H. Cordier, 1913-1916. -.. Cathay and the Way thither (Hakluyt Society), London, 4 vol. 